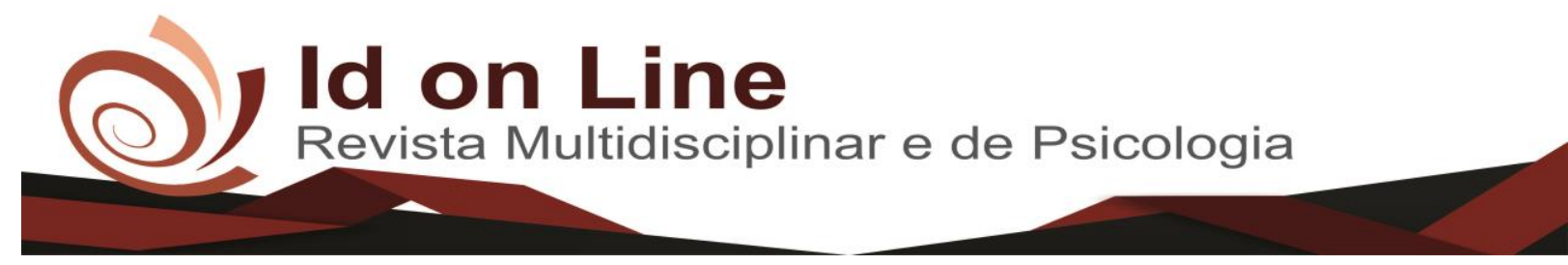

Artigo

\title{
Avaliação da estatura em crianças nascidas com baixos níveis de vitamina $D$
}

\author{
Franciane Silva Luiz', Mara Rúbia Maciel Cardoso do Prado ${ }^{2}$, Pedro Paulo do Prado Júnior ${ }^{3}$, \\ Lorrane Stéfany Ribeiro Assunção ${ }^{4}$, Fernanda Barbieri Boro ${ }^{5}$, Fábio da Costa Carbogim 6
}

\begin{abstract}
Resumo: Objetivo: avaliar o crescimento de crianças nascidas com baixos níveis de vitamina D. Método: Pesquisa de delineamento transversal, parte de um estudo de coorte. Avaliou-se 20 crianças de 4 anos de idade cujas mães aceitaram a realização da visita domiciliar. Utilizou-se análise descritiva para avaliar as variáveis independentes e análise de Correlação de Pearson para avaliar a influência da hipovitaminose D das crianças no nascimento em relação ao crescimento. Resultados: Houve prevalência de hipovitaminose D em crianças do sexo feminino, não brancas, expostas à luz solar, com renda insuficiente, que tiveram aleitamento materno exclusivo, mas que não foram suplementadas com vitamina D. O crescimento das crianças e a hipovitaminose D no nascimento não estiveram correlacionados. Conclusão: Enfatiza-se a importância das orientações em relação à exposição solar e suplementação de vitamina $\mathrm{D}$, bem como a necessidade de se criar protocolos que resguardem as intervenções do profissional quanto à suplementação com VD.
\end{abstract}

Palavras Chaves: Saúde da criança; Estatura; Vitamina D.

\section{Assessment of height in children born with low levels of D vitamin}

Abstract: The aim of the study was to evaluate the growth of children born with low levels of D vitamin. The method is Cross-sectional design study, part of a cohort study. A total of 204 -year-old children whose mothers accepted the home visit were evaluated. Descriptive analysis was used to evaluate the independent variables and Pearson's correlation analysis to evaluate the influence of hypovitaminosis D at birth on growth. As the results showed, there was a prevalence of hypovitaminosis D in non-white, sun-exposed, low-income children who had exclusive breastfeeding but who were not supplemented with D vitamin . Children's growth and hypovitaminosis $\mathrm{D}$ at birth were not correlated. It can be concluded that is emphasized the importance of the guidelines in relation to sun exposure and D vitamin supplementation, as well as the need to create protocols that safeguard the professional's interventions regarding RV supplementation.

Keywords: Child health; Stature; D vitamin.

\footnotetext{
${ }^{1}$ Enfermeira. Mestranda em Enfermagem. Universidade Federal de Juiz de Fora. Juiz de Fora; Minas Gerais, Brasil.

E-mail: franciane.silva.192@gmail.com.

${ }^{2}$ Enfermeira. Doutora em Ciência da Nutrição. Universidade Federal de Viçosa. Viçosa; Minas Gerais, Brasil.

E-mail: enfermeiramara@hotmail.com.

${ }^{3}$ Enfermeiro. Doutor em Ciência da Nutrição. Universidade Federal de Viçosa. Viçosa; Minas Gerais, Brasil.

E-mail: pedro.prado@ufv.br.

${ }^{4}$ Enfermeira. Universidade Federal de Viçosa. Viçosa; Minas Gerais, Brasil. E-mail: lolo_stefany@ hotmail.com

${ }^{5}$ Enfermeira. Universidade Federal de Viçosa. Viçosa; Minas Gerais, Brasil. E-mail: fernanda.boro@ufv.br

${ }^{6}$ Enfermeiro. Doutor em Ciências. Universidade Federal de Juiz de Fora. Juiz de Fora, Minas Gerais, Brasil. E-mail: fabiocarbogim@gmail.com.

Autor correspondente: Franciane Silva Luiz; Faculdade de Enfermagem. Rua José Lourenço Kelmer - São Pedro, Juiz de Fora - MG, Brasil, 36036-900. Contato: franciane.silva.192@gmail.com.
} 


\section{Introdução}

O crescimento é um excelente indicador do estado de higidez da criança, representando no Brasil, o déficit antropométrico mais importante, devido à sua intensa relação com as condições de vida intrauterina e fatores ambientais, como alimentação, higiene, habitação, saúde e cuidados gerais, sendo a nutrição inadequada a principal responsável pelo déficit na estatura. Diante o exposto, o Ministério de Saúde prioriza a monitorização do crescimento, desde o nascimento até os dez anos de idade, visando a identificação precoce de alterações e consequentes complicações (BRASIL, 2012).

Nesse contexto, o papel que a Vitamina D (VD) desempenha no corpo tem se tornado temática de interesse para pesquisas, pois a VD, por estar envolvida no crescimento esquelético, torna-se essencial durante a infância e a adolescência. Isso porque, níveis plasmáticos normais desse hormônio promovem a absorção de $30 \%$ do cálcio dietético e mais de $60-80 \%$ em períodos de crescimento, devido à alta demanda de cálcio. Dessa forma, durante a infância, a Deficiência de VD (DVD) pode causar retardo de crescimento, anormalidades ósseas e aumento do risco de fraturas na vida adulta. Dentre as consequências mais comuns da DVD, estão as deformidades ósseas, caracterizadas na infância pelo raquitismo, doença que resulta em baixa estatura, fraqueza muscular, deformidade esquelética, hipocalcemia e tetania (HOLICK, 2006; BUENO; CZEPIELLEWSKI, 2008; JUNIOR et al, 2011; MAEDA et al, 2014).

Apesar de sua iminente relação com o crescimento infantil, a hipovitaminose D (insuficiência ou deficiência de Vitamina D) é altamente prevalente e constitui um problema de saúde pública em todo o mundo, com destaque para a Europa, África, América do Norte, Oriente Médio e alguns países da América do Sul, como Chile e Argentina. Estima-se que 1 bilhão de pessoas tem deficiência ou insuficiência de Vitamina D (VD) mundialmente. No Brasil a hipovitaminose D ocorre em várias faixas etárias e apesar das particularidades climáticas de cada região, os brasileiros apresentam baixa ingestão de VD. (JUNIOR et al, 2011; MAEDA et al, 2014; WALCKER; HOLLICK, 2013).

Vários fatores levam à insuficiência de VD, dentre estes estão: a menor incidência de radiação solar, que varia com a latitude e com a estação do ano; a obesidade; o uso de protetor solar; os hábitos culturais; a gravidez; o aleitamento materno; o tipo de pele; o envelhecimento; o uso de medicações que interferem no metabolismo da VD (anticonvulsivantes, glicocorticoides, antifúngicos, antirretrovirais, colestiramina); e portadores de doenças que 
alteram o metabolismo da 25(OH)D como fibrose cística, doenças do trato gastrintestinal, hematológicas, renais, insuficiência cardíaca e imobilização (JUNIOR et al, 2011; KHAZAI; TANGPRICHA, 2008;MAEDA et al, 2014; PAPPA et al, 2012; PREMAOR; FURLANETTO, 2006).

Atentar-se aos fatores supracitados é essencial para prestar uma assistência de qualidade com vista a reduzir a morbimortalidade infantil ocasionadas pela DVD.

Destarte, tendo em vista a escassez de pesquisas relacionadas à hipovitaminose $\mathrm{D}$, principalmente na faixa etária compreendida nesse estudo, bem como o recente interesse no papel que a VD desempenha no corpo, os efeitos da sua deficiência e que os problemas de crescimento frequentemente começam na vida intrauterina e continuam até os dois primeiros anos de vida, períodos que representam as melhores chances relacionadas com a prevenção do déficit de altura (JUNIOR et al, 2011; BENTLEY, 2015; PEDRAZA et al 2013) é que o objetivo deste trabalho foi avaliar o crescimento de crianças nascidas com baixos níveis de vitamina D.

\section{Método}

Pesquisa de delineamento transversal integrante de um estudo de coorte intitulado "Fatores associados aos níveis de vitamina D do binômio mãe-filho ao nascimento e aos seis meses de vida", que foi aprovado pelo Comitê de Ética em Pesquisa com Seres Humanos da Universidade Federal de Viçosa pelo protocolo $n^{\circ}$ 51/2012. Na coorte, foram analisadas 65 crianças nascidas no município de Viçosa-MG.

Na busca de avaliar as intervenções realizadas no estudo de coorte decidiu-se por avaliar as crianças aos 4 anos de idade. Dessa forma, incluíram-se no estudo, crianças cujas mães assinaram o Termo de Consentimento Livre e Esclarecido e aceitaram a realização da visita domiciliar para aplicação do questionário e a avaliação antropométrica da criança, chegando assim a um "n" de 20 crianças. As perdas ocorreram devido a alguns fatores, entre eles mudança de endereço, troca do número do telefone, a recusa das mães em permitir a avaliação antropométrica da criança e a aplicação do questionário.

Os dados socioeconômicos (sexo, raça e renda familiar) e biológicos (aleitamento materno, exposição solar, uso de protetor e de suplementação com VD) foram conseguidos através de questionário aplicado às mães durantes as vistas domiciliares. 
A cor da pele das crianças foi autorrelatada durante a aplicação do questionário pelas mães e confirmada na Declaração de Nascidos pelos pesquisadores, sendo categorizada em branca e não branca. Para classificação da renda familiar, também considerou-se a informação autorrelatada das mães, sendo classificadas em suficiente e não suficiente. De acordo com o tempo de exposição ao sol a amostra foi dividida em dois grupos: adequado >30 minutos/semana ou inadequado $\leq 30$ minutos/semana (MATTOS et al, 2012). Conforme os níveis de VD das crianças avaliadas no nascimento (sangue do cordão umbilical) a amostra foi dividida entre suficiência e hipovitaminose D (insuficiência e deficiência de VD). A DVD em crianças foi definida como $25(\mathrm{OH}) \mathrm{D}<20 \mathrm{ng} / \mathrm{mL}$, a insuficiência de $25(\mathrm{OH}) \mathrm{D}$ entre $\geq 20$ e $<30$ $\mathrm{ng} / \mathrm{mL}$ e a suficiência de 25(OH)D $\geq 30 \mathrm{ng} / \mathrm{Ml}$ (MAEDA et al, 2014; PREMAOR; FURLANETTO, 2006; WALCKER; HOLLICK, 2013; COLE et al, 2010).

Os dados de peso e estatura foram convertidos nos índices peso/idade(P/I), peso/estatura $(\mathrm{P} / \mathrm{E})$, estatura/idade (E/I), em escore-Z, empregando-se como referência o padrão antropométrico proposto pela Organização Mundial de Saúde (WHO, 2009). As variáveis antropométricas foram classificadas utilizando-se o programa Anthroplus.

A variável desfecho ou dependente do estudo foi o atraso no crescimento em crianças com hipovitaminose D. As variáveis independentes foram: condições socioeconômicas da criança e de saúde infantil na idade atual (incluindo a exposição solar da criança em minutos, uso de protetor solar, suplementação com vitamina D).

O banco de dados foi elaborado com dupla digitação, no Microsoft Office Excel 2007 e para as análises estatísticas utilizou-se o programa Statical Package for the Social Sciences (SPSS) versão 20.0.

Realizou-se estatística descritiva, sendo verificada a frequência simples e relativa, média e desvio-padrão das variáveis categóricas e continuas, respectivamente. O teste de Kolmogorov-Smirnov foi utilizado para avaliar normalidade no caso das variáveis quantitativas.

Foi utilizado análise de Correlação de Pearson a fim de avaliar se houve influência da hipovitaminose D das crianças no nascimento em relação ao crescimento delas na idade atual (4 anos). O nível de significância estatística adotado foi $\alpha<5 \%$. 


\section{Resultados}

Das crianças avaliadas, observa-se na Tabela 1 que a maioria, $12(60 \%)$ eram do sexo feminino; 12(60\%) foram autorrelatadas não branca; 18 (90\%) tiveram-aleitamento materno exclusivo; 15 (75\%) não foram suplementadas com VD; 14 (70\%) eram expostas à luz sol; 10 (50\%) faziam uso de protetor solar; e $14(70 \%)$ das mães relataram que a renda familiar era insuficiente. Não houve associação entre os níveis de VD dos RN e as variáveis independentes, sexo, raça, aleitamento materno, suplementação com VD, exposição solar, protetor solar e renda autorrelatada.

Tabela 1: Descrição das variáveis sociodemográficas e biológicas das crianças com 4 anos atualmente que apresentaram hipovitaminose $\mathrm{D}$ ao nascimento.

\begin{tabular}{lll} 
& N & $\%$ \\
\hline Sexo & 8 & \\
M & 12 & 40 \\
F & 8 & \\
Raça & 12 & 40 \\
Branca & & 60 \\
Não Branca & 18 & \\
Aleitamento Materno & 2 & 90 \\
Exclusivo & & 10 \\
Não exclusivo & 5 & \\
Suplementação com VD & 15 & 25 \\
Sim & 14 & 75 \\
Não & 6 & 70 \\
Exposição Solar & & 30 \\
Sim & 10 & 50 \\
Não & 10 & 50 \\
Protetor Solar & & \\
Atende & 6 & 70 \\
Não atende & 14 & \\
Renda autorrelatada & & \\
Suficiente & Insuficiente &
\end{tabular}

Fonte: Os autores (2016)

$\mathrm{Na}$ tabela 2, pode-se observar que as médias de peso $(\mathrm{g})$, altura $(\mathrm{cm})$ e IMC foram de 16275 gramas $\pm 3405 \mathrm{~g}, 102 \mathrm{~cm} \pm 5 \mathrm{~cm}$ e $15,49 \mathrm{~kg} / \mathrm{m}^{2} \pm 2,1413 \mathrm{~kg} / \mathrm{m}^{2}$, respectivamente. Os valores mínimo e máximo para peso foram 11000 gramas e 26200 gramas, para altura $(\mathrm{cm}) 89$ e 110 e para o IMC $11,80 \mathrm{~kg} / \mathrm{m}^{2}$ e $21,50 \mathrm{~kg} / \mathrm{m}^{2}$, respectivamente. Neste ponto, evidenciou-se duas 
crianças abaixo (Score $Z=-2,85$ e -2,43) e outras duas acima (Score $Z=2,16$ e 3,18) do peso, uma criança de baixa estatura (Score $Z=-3,01)$ e duas acima (Score $Z=2,03$ e 2,08) e duas crianças com IMC abaixo (Score $Z=-2,99$ e -2,72) e uma acima (Score $Z=3,84$ ) dos pontos de corte (entre -2 e +2 ) de Score $Z$ de peso, altura e IMC. Não houve associação entre os níveis de VD dos RN em relação ao peso, a altura e o IMC.

Neste contexto, evidenciou-se que a criança que apresentou baixa estatura nesse estudo era do sexo feminino, da raça não branca, teve aleitamento materno exclusivo, não foi suplementada com VD, era exposta a luz solar por tempo menor ou igual a 30 minutos, fazia uso de protetor solar e renda autorrelatada não suficiente.

Com relação às duas crianças com IMC abaixo do valor de referência, uma era do sexo feminino, uma das crianças foi exposta a luz solar por tempo menor ou igual a $30 \mathrm{~min}$ (sexo masculino), ambas da raça não branca, tiveram aleitamento materno exclusivo, não foram suplementadas com VD, não faziam uso de protetor solar e tiveram renda autorrelatada não suficiente. Já a criança com IMC acima do valor de referência é do sexo masculino, da raça não branca, teve aleitamento materno exclusivo, foi suplementada com VD, era exposta a luz solar por tempo menor ou igual a $30 \mathrm{~min}$, fazia uso de protetor solar e renda autorrelatada suficiente.

Tabela 2: Variáveis antropométricas das crianças com 4 anos com hipovitaminose D ao nascimento.

\begin{tabular}{|c|c|c|c|c|}
\hline & Média & $\begin{array}{l}\text { Desvio Padrão } \\
(+/-)\end{array}$ & Mínimo & Máximo \\
\hline Peso (g) & 16275 & 3405 & 11000 & 26200 \\
\hline Score z peso & 0,0690 & 1,4377 & $-2,85$ & 3,18 \\
\hline Altura (cm) & 102 & 5 & 89 & 110 \\
\hline Score z altura & 0,138 & 1,139 & $-3,010$ & 2,08 \\
\hline $\mathrm{IMC}\left(\mathrm{Kg} / \mathrm{m}^{2}\right)$ & 15,49 & 2,1413 & 11,80 & 21,50 \\
\hline Score z IMC & $-0,0255$ & 1,5773 & $-2,99$ & 3,84 \\
\hline
\end{tabular}

Fonte: Os autores (2016)

Observa-se no gráfico 1 a relação entre o nível plasmático de VD da criança ao nascer e a altura $(\mathrm{cm})$ da criança aos 4 anos de idade. Foram avaliadas 20 crianças com hipovitaminose $\mathrm{D}$ ao nascer, das quais apenas uma apresentou retardo no crescimento (Score $z=-3,01$ ). Visto que o " $r$ " encontrado foi de -0,295, constatou-se uma correlação linear fraca, ou seja, o crescimento na idade atual das crianças e a DVD no nascimento não estiveram correlacionados. 
Gráfico 1: Análise de correlação do crescimento das crianças que apresentaram hipovitaminose D no nascimento.

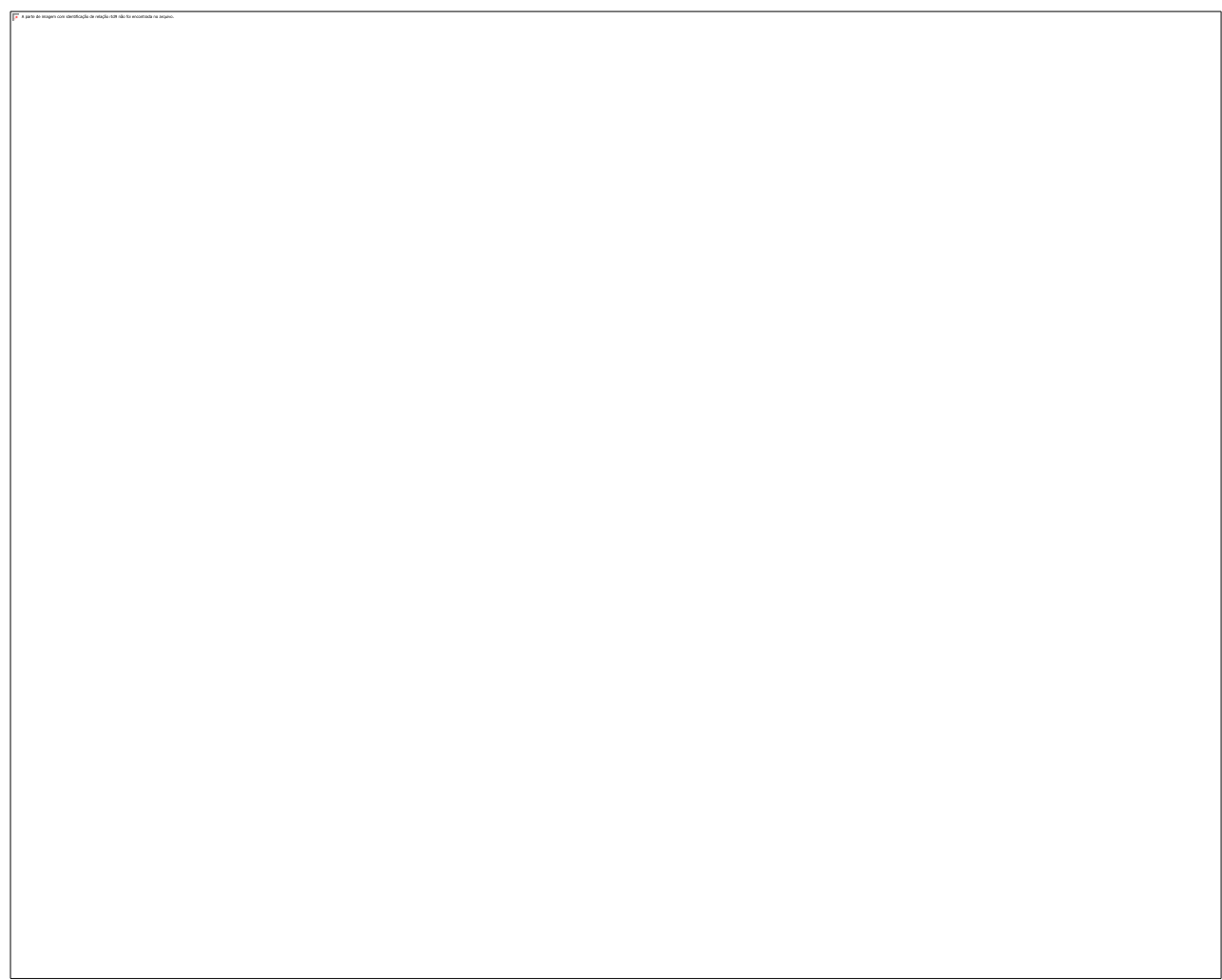

Observação: $r=-0,295 \mathrm{p}=0,20$

\section{Discussão}

A DVD é um problema de saúde pública no mundo, e o Brasil insere-se nesse contexto representado pelas diversas faixas etárias (JUNIOR et al, 2011; MAEDA et al, 2014). Na tabela 1 foram descritos alguns fatores de risco relacionados com a ocorrência dos baixos níveis de VD e por consequente com o crescimento infantil inadequado. Encontrou-se uma maior prevalência de hipovitaminose $\mathrm{D}$ em crianças do sexo feminino, o que pode ser justificado pela composição da amostra, majoritariamente feminina (60\%). Com relação ao sexo, outras pesquisas apontam que mulheres são mais capazes de produzir VD do que os homens, pois a maioria delas são brancas, quando comparadas ao sexo masculino. Como será discutido a seguir, pessoas não brancas possuem maior dificuldade em produzir essa vitamina (WALCKER; HOLLICK, 2013). Ressalta-se ainda a necessidade de se promover investigações 
científicas sobre a prevalência de hipovitaminose D em relação ao sexo na faixa etária compreendida nesse estudo.

No que se refere à raça, observa-se nesse estudo uma maior prevalência de hipovitaminose $\mathrm{D}$ em pessoas não-brancas, fato esse registrado em outros estudos e que ocorre, pois a maior concentração de melanina nesse público atua como uma barreira para a radiação Ultravioleta B (UVB). Assim, tendo em vista que a melanina absorve a radiação UVB, pessoas não brancas precisam de um tempo de exposição de 3 a 5 vezes maior (JUNIOR et al, 2011; URRUTIA-PEREIRA; SOLÉ, 2015).

No que concerne à renda, evidencia-se que a maioria relatou ser insuficiente, o que impacta diretamente na ingestão alimentar. Sabe-se que uma dieta saudável auxilia na manutenção dos níveis plasmáticos de VD. No entanto, os alimentos mais acessíveis, fonte dessa vitamina são poucos consumidos devido ao elevado conteúdo de colesterol, como a gema de ovo, fígado, manteiga e leite. Outros alimentos ricos em óleos de fígado de peixes como atum e linguado, bacalhau e peixes como salmão, cavala, sardinha, enguia, arenque e atum também são ricos em VD (BUENO; CZEPIELEWSKI, 2008), porém são produtos de maior custo e não fazem parte da realidade da maioria dos participantes.

Os primeiros anos de vida de uma criança são caracterizados por crescimento acelerado e enormes aquisições no processo de desenvolvimento, dessa forma, torna-se indiscutível a importância da alimentação da criança nesse período (BRASIL, 2010). Nesse contexto, o aleitamento materno é um relevante fator na redução dos índices de morbimortalidade infantil e no incremento da saúde da criança, tendo em vista seu significativo valor nutricional e imunológico, além de seus impactos na redução da probabilidade da manifestação de problemas alérgicos, respiratórios, obesidade, hipertensão arterial, hipercolesterolemia e diabetes mellitus (KALIL; AGUIAR, 2016). Recomenda-se assim o Aleitamento Materno Exclusivo até os seis meses de idade e a introdução da alimentação complementar a partir dessa fase, com a manutenção do aleitamento materno por dois anos ou mais (SALDAN et al, 2015).

No entanto, com relação à VD sabe-se que leite materno apresenta quantidade insuficiente desse hormônio, portanto, os RN em amamentação exclusiva possuem alto risco para desenvolverem hipovitaminose D (URRUTIA-PEREIRA; SOLÉ, 2015). Além disso, observa-se um aumento no índice de RN com hipovitaminose D devido à alta DVD materna (PRADO et al, 2015). Nesta perspectiva, para prevenir a hipovitaminose D, as Academias Mineira e Brasileira de Pediatria, em Abril de 2012, e a Sociedade Brasileira de Endocrinologia 
e Metabologia em 2014, baseadas no Institute of Medicine recomendam que durante o primeiro ano de vida os bebês recebam imediatamente complementação diária de 400 UI de VD; entre 1 e 70 anos (600 UI); e após 70 anos (800 UI) (KHAZAI; TANGPRICHA, 2008; HOSSEINNEZHAD; HOLICK, 2013). ). Portanto, ressalta-se a necessidade de se iniciar a suplementação com VD materno-infantil.

As fontes de VD são a exposição solar, a dieta e a suplementação. Sabe-se que a primeira é a principal fonte para manutenção dos níveis de VD, devido à alta dependência entre a produção cutânea e a exposição solar. Assim, pessoas com baixa insolação constituem a principal população de deficientes de VD (MAEDA, et al, 2014). Recomenda-se que crianças menores de seis meses de idade sejam expostas diretamente à luz solar a partir dos 14 dias de vida durante 30 minutos/semana usando apenas fraldas, ou seja, orienta-se a exposição solar de 6 a 8 minutos por dia, três vezes por semana ou de duas horas/semana em exposição parcial, sendo assim 17 minutos por dia com exposição da face e das mãos. Dessa forma, a exposição solar é uma das principais formas de se prevenir o raquitismo (MATTOS et al, 2012; WALKER; HOLLICK, 2013).

Observa-se que 50\% da amostra utilizou protetor solar, no entanto, o uso desse produto com fator de proteção 30 diminui a produção de VD na pele em aproximadamente $95 \%$ dos casos URRUTIA-PEREIRA; SOLÉ, 2015). Nesta perspectiva, orienta-se que durante a exposição solar para síntese de VD não seja utilizado protetores solar. Destaca-se também a importância do uso desse produto nos demais momentos para a prevenção do câncer de pele.

Com relação à tabela 2, no que diz respeito aos valores de referência nos extremos dos Score $Z$ de peso, altura e IMC, evidencia-se a necessidade de se realizar orientações aos cuidadores e familiares para que as crianças em questão atinjam os valores adequados para sua idade e altura. Dessa forma, contribuirá para a prevenção de doenças e agravos e promoção da saúde. Com relação ao peso, sabe-se que a obesidade é associada à DVD, pois a VD, lipossolúvel, é detida pela gordura corporal (URRUTIA-PEREIRA; SOLÉ, 2015; MAEDA et al, 2014). Estudos revelam que a hipovitaminose D pode prognosticar uma aceleração no aumento da massa gorda e, por consequente um aumento na incidência da obesidade. Isso se dá, porque provavelmente a VD insere-se no mecanismo que regula a apoptose dos adipócitos. Assim, destaca-se as complicações da desnutrição infantil, atentando-se para os impactos do baixo peso e do sobrepeso na vida infantil e adulta. Nesta perspectiva, estudos que abarquem a 
suplementação com VD em humanos tornam-se necessários para avaliar sua eficácia no combate a obesidade (MAEDA et al, 2014).

Em relação à altura, deve-se atentar para a baixa estatura, visto que o crescimento é um indicador significativo do estado de saúde da criança. Sabe-se ainda que a VD exerce papel fundamental no metabolismo ósseo, agindo na absorção do cálcio pelo intestino e na modulação da secreção de PTH e participando da função muscular e das células ósseas (MAEDA et al, 2014; KHAZAI; TANGPRICHA, 2008). Trata-se de um hormônio que regula o metabolismo do cálcio e do fósforo e estimula a diferenciação dos condrócitos da placa de crescimento, sendo, portanto, relevante para a constituição dos dentes, formação e crescimento dos ossos (BUENO; CZEPIELEWSKI, 2008; BUENO; CZEPIE, 2007).

Por fim, entende-se que a correlação linear existente entre a altura da criança aos 4 anos e o nível plasmático de VD ao nascer foi fraca. No entanto, o valor de "p" pode ter sido influenciado pelo baixo número de participantes, destacando-se, portanto, que a relação entre as variáveis analisadas pode ser positiva ao se analisar uma amostra maior.

\section{Conclusão}

Apesar de não ter sido encontrado associação entre o crescimento na idade atual das crianças e a DVD no nascimento neste estudo, cabe hastear a relação da VD com o crescimento infantil, bem como a necessidade de outras pesquisas que estudem o fenômeno aqui apresentado. Isso porque, os resultados podem apresentar correlação positiva entre o crescimento e a VD em amostras maiores.

A premissa anterior é justificada ao se compreender a VD como um hormônio que exerce várias funções no organismo, sendo essencial que os indivíduos mantenham os níveis plasmáticos dessa vitamina suficientes a fim de contribuir para a manutenção de uma vida hígida e de qualidade. Para tanto, a profilaxia deve ser iniciada desde os primeiros dias de vida da criança tendo em vista os benefícios referentes à prevenção de doenças e agravos nessa fase, principalmente relacionado a um relevante indicador de saúde infantil, o crescimento.

Ademais, os níveis plasmáticos de VD impactam diretamente no processo de crescimento ósseo da criança e se relaciona à fatores modificáveis e não modificáveis. Nesta perspectiva, destaca-se a importância das orientações dos profissionais de saúde de forma 
multiprofissional, envolvendo enfermeiros, médicos, assistentes sociais e nutricionistas. Outrossim, atenta-se para a criação de protocolos de saúde da criança que resguardem as intervenções do profissional quanto à suplementação com VD.

Destaca-se a importância de ações estratégicas relacionadas às variáveis modificáveis como renda familiar, aleitamento materno, exposição solar, uso de protetor e de suplementação de vitamina $\mathrm{D}$, tendo em vista que as mesmas quando favoráveis à criança impactam positivamente no crescimento ósseo, prevenindo-se complicações decorrentes no retardo do crescimento infantil, como o raquitismo na faixa etária em questão.

Portanto, a fim de se prevenir um desequilíbrio no processo saúde-doença causadas pela hipovitaminose $\mathrm{D}$ enfatiza-se o acompanhamento de forma longitudinal e resolutiva da criança por toda a equipe de saúde e independente do nível de atenção. Diante desse contexto, surge, pois, um novo cenário em que a equipe de saúde pode atuar para promover a saúde e prevenir doenças e agravos em crianças, contribuindo para a redução da morbimortalidade no público alvo em questão.

\section{Referências}

BENTLEY, J. The role of vitamin D in infants, children and young people. Nursing Children and Young People, v. 27, n. 1, p. 28, 2015.

BRASIL. DEPARTAMENTO DE ATENÇÃO BÁSICA, SECRETARIA DE ATENÇÃO À SAÚDE, MINISTÉRIO DA SAÚDE. Dez passos para uma alimentação saudável: guia alimentar para crianças menores de dois anos: um guia para o profissional da saúde na atenção básica. 2010. Disponível em: http://www.redeblh.fiocruz.br/media/10palimsa_guia13.pdf

BRASIL. Ministério da Saúde. Secretaria de Atenção à Saúde. Departamento de Atenção Básica. Saúde da Criança: Crescimento e Desenvolvimento-Caderno de Atenção Básica nº33. Brasília. Editora do Ministério da Saúde, 2012. Disponível em: http://bvsms.saude.gov.br/bvs/publicacoes/saude_crianca_crescimento_desenvolvimento.pdf

BUENO, A.L.; CZEPIELEWSKI, M.A. A importância do consumo dietético de cálcio e vitamina $D$ no crescimento. Jornal de pediatria. Rio de Janeiro. Vol. 84, n. 5,(Set. 2008), p. 386-394, 2008. Disponível em: http://www.scielo.br/scielo.php?pid=S0021$75572008000600003 \&$ script $=$ sci_abstract\&tlng=pt

BUENO, A.L.; CZEPIELEWSKI, M.A. Micronutrientes envolvidos no crescimento. Revista HCPA. Porto Alegre. Vol. 27, n. 3,(2007), p. 47-56, 2007. Disponível em: 
http://www.nutricaoemfoco.com.br/NetManager/documentos/micronutrientes_envolvidos_no _crescimento.pdf

COLE, C.R. et al. 25-hydroxyvitamin D status of healthy, low-income, minority children in Atlanta, Georgia. Pediatrics, p. peds. 2009-1928, 2010.

HOLICK, M.F. Resurrection of vitamin D deficiency and rickets. The Journal of clinical investigation, v. 116, n. 8, p. 2062-2072, 2006.

HOSSEIN-NEZHAD, A.; HOLICK, M.F. Vitamin D for health: a global perspective. Mayo Clin Proc 2013; 88:720-55.

JUNIOR, E.P.S. et al. Epidemiologia da deficiência de vitamina D. Revista Científica do ITPAC 2011; 4(3). Disponível em: https://assets.itpac.br/arquivos/Revista/43/2.pdf

KALIL, I.R.; AGUIAR, A.C. Trabalho feminino, políticas familiares e discursos próaleitamento materno: avanços e desafios à equidade de gênero. Saúde em debate, v. 40, p. 208223, 2016. Disponível em: http://www.scielo.br/scielo.php?pid=S0103$11042016000300208 \&$ script $=$ sci_abstract\&tlng=pt

KHAZAI, N.; JUDD, S.E.; TANGPRICHA, V. Calcium and vitamin D: skeletal and extraskeletal health. Current rheumatology reports, v. 10, n. 2, p. 110-117, 2008.

MAEDA, S.S. et al. Recomendações da Sociedade Brasileira de Endocrinologia e Metabologia (SBEM) para o diagnóstico e tratamento da hipovitaminose D. Arquivos Brasileiros de Endocrinologia \& Metabologia, 2014. Disponível em: http://www.scielo.br/scielo.php?pid=S0004$27302014000500411 \&$ script=sci_abstract\&tlng=pt

MATTOS, A. P.; BRASIL, A. L. D.; MELLO, E. D. Manual de Orientação: alimentação do lactente, alimentação do pré-escolar, alimentação do escolar, alimentação do adolescente, alimentação na escola. Sociedade Brasileira de Pediatria. Departamento de Nutrologia, São Paulo, p. 32-38, $2006 . \quad$ Disponível em: http://www.sbp.com.br/fileadmin/user_upload/pdfs/14617a-pdmanualnutrologiaalimentacao.pdf

NEZHAD, A. HOLICK, M.F. Vitamin D for health: a global perspective. Mayo Clin Proc 2013; 88:720-55.

PAPPA, H.M. et al. Treatment of vitamin D insufficiency in children and adolescents with inflammatory bowel disease: a randomized clinical trial comparing three regimens. The Journal of Clinical Endocrinology \& Metabolism, v. 97, n. 6, p. 2134-2142, 2012.

PEDRAZA, D.F.; ROCHA, A.C.D.; SOUSA, C.P.C. Crescimento e deficiências de micronutrientes: perfil das crianças assistidas no núcleo de creches do governo da Paraíba, Brasil. Ciência \& Saúde Coletiva, v. 18, p. 3379-3390, 2013. Disponível em: http://www.scielo.br/scielo.php?script=sci_arttext\&pid=S1413-81232013001100027 
PRADO, M.R.M.C. et al. Prevalência de deficiência de vitamina D e fatores associados em mulheres e seus recém-nascidos no período pós-parto. Revista Paulista de Pediatria, v. 33, n. 3, 2015. Disponível em: http://www.scielo.br/scielo.php?pid=S010305822015000300286\&script=sci_abstract\&tlng=pt

PREMAOR, M.O.; FURLANETTO, T.W. Hipovitaminose D em adultos: entendendo melhor a apresentação de uma velha doença. Arquivos brasileiros de endocrinologia \& metabologia $=$ Brazilian archives of endocrinology and metabolism. São Paulo. Vol. 50, n. 1 (fev. 2006), p. 25-37, 2006.

SALDAN, P.C. et al. Práticas de aleitamento materno de crianças menores de dois anos de idade com base em indicadores da Organização Mundial da Saúde. Revista de Nutrição, v. 28, n. 4, p. 409-420, 2015.

URRUTIA-PEREIRA, M.; SOLÉ, D. Deficiência de vitamina D na gravidez e o seu impacto sobre o feto, o recém-nascido e na infância. Revista Paulista de Pediatria, v. 33, n. 1, p. 104113, 2015. Disponível em: http://www.scielo.br/pdf/rpp/v33n1/pt_0103-0582-rpp-33-0100104.pdf

WHO; WORLD HEALTH ORGANIZATION. NUTRITION FOR HEALTH. WHO child growth standards: growth velocity based on weight, length and head circumference: methods and development. World Health Organization, 2009.

Como citar este artigo (Formato ABNT):

LUIZ, Franciane Silva; PRADO, Mara Rúbia Maciel Cardoso do; PRADO JÚNIOR, Pedro Paulo do; ASSUNÇÃO, Lorrane Stéfany Ribeiro; BORO, Fernanda Barbieri; CARBOGIM, Fábio da Costa. Avaliação da estatura em crianças nascidas com baixos níveis de vitamina D. Id on Line Rev.Mult. Psic., 2018, vol.12, n.42, p. 295-307. ISSN: 1981-1179.

Recebido: 30/08/2018;

Aceito: 10/09/2018 